\title{
Proposal of a Knowledge-Based Engineering Methodology for Mass Customization
}

\author{
Kiyan Vadoudi ${ }^{1}$ and Sergio Terzi ${ }^{2}$ \\ ${ }^{1}$ Politecnico di Milano, Milano, Italy \\ ${ }^{2}$ University of Bergamo, Bergamo, Italy \\ kiyan.vadoudi@mail.polimi.it, sergio.terzi@unibg.it
}

\begin{abstract}
The proposed methodology aims to tackle Mass Customization (MC) from a knowledge management perspective and attempts to propose a systematic framework to implement and modify the process of MC, based on best practice with storing the data as experiences, customer behaviors and data related to a specific product family. This process is the result of providing support and appropriate automation of repetitive and routine design tasks to perform multidisciplinary activities. The forces of the proposed methodology are its ease to use, providing a consistency process of developing and maintaining Knowledge-Based Engineering (KBE) methodology for implementing MC and reduction in leadtime and cost in the design process. A first tentative application made in the apparel industry of the methodology is also reported.
\end{abstract}

Keywords: Knowledge management, Knowledge-Based Engineering, Mass Customization.

\section{Introduction}

In today's rapidly changing and globalizing markets the traditional mass production paradigm is not possible to support high variety of customer needs. In this context, Mass Customization (MC) [1],[2] appeared as a new paradigm representing the trend towards the production of highly variant products under mass production time and cost conditions. Key enablers for implementing a MC strategy [3], from one side are advanced manufacturing systems - which allows the provision of customer-individual products using flexible production processes - and on the other side is the availability of rapid design and development processes for creating highly product and service variants.

In this context, this paper proposes a methodology to tackle MC from a knowledge management perspective for reducing lead-times and costs in the design process. A first tentative application made in the apparel industry is also reported.

\section{State of the Art}

The nature of MC occurs in the product and service and the challenge is the ability to realize and capture hidden market niches and subsequently develop technical 
capabilities to meet the different types of needs for target customers. Understanding the latent market niches requires the exploration of customer needs. To encapsulate the needs of target customer groups means to imitate existing or potential competitors in quality, cost and quick response. Thus, the requirements of MC depend on a balance of three elements: (i) time-to-market / quick responsiveness, (ii) variety / customization and (iii) economy of scale for volume production efficiency [4].

The main stress of design for $\mathrm{MC}$ is to elevate the practice of designing individual products to design product families [4]. A product family is a set of products derived from a common platform which includes a set of variables, features or components that remain constant in a product platform / module and from product to product [5]. A modular design approach gives the capability to rapidly create a large number of product variants, generating a lot of diversified knowledge in short times.

For keeping the MC constrains, design knowledge should be managed in an efficient way, avoiding redundant design tasks and supporting the process of the knowledge creation, management, extraction and re-use. Knowledge management has a long history and related to World War II in order to build the fighter planes. That time, observes shown that building of second airplane took less time and less defects than the first one and this understanding about process of production was beginning to appear the concept of knowledge management. By the mid-1980s, the importance of knowledge as a competitive asset was apparent and by increasing the importance of organization's knowledge [6],[7], the concern emerged about dealing by growing up by the amount of available knowledge in organization.

The computer technology that cooperated so heavily to superabundance of information started to become part of the solution, in a variety of domains. nowledgeBased Engineering (KBE) is a combination of object-oriented programming, artificial intelligence, and computer aided design [8] in order to capture product and process information to allow businesses to model engineering processes, and then use the model to automate all or part of the process.

A number of methodologies are available to support the development of $\mathrm{KBE}$ applications and systems [9]. By far the most famous of these is the Methodology and software tools Oriented to Knowledge-based engineering Applications, or MOKA methodology [10],[11]. This methodology consists of six life-cycle steps (Identify, Justify, Capture, Formalize, Activate and Delivery), and it is supported by informal and formal models to take a project from beginning towards industrialization and actual use. KNOMAD (Knowledge Nurture for Optimal Multidisciplinary Analysis and Design) is another methodology to address the identified shortcomings of $\mathrm{KBE}$ [12], consisting of six steps: Knowledge Capture, Normalization, Organization, Modeling, Analysis and Delivery.

\section{The Proposed Methodology}

The global competitions in industries change manufactures' view in terms of contact with customer from a seller point of view to a buyer. Both changes from companies' side and changes in customers' needs, result in a drastic increase in the number of 
product variants and costs of production. Through these changes, companies are trying to stay in competitive atmosphere through making a modeled system for their production processes by introducing the platform concept [13], which changed production concept from no customizable products to modular products. Moreover, by recent development of IT technologies the possibility of using software-based product configuration systems [14] increased. These systems are supporting the process of customized product development by using the modules based on the customer's requirements. The proposed methodology uses the platform concept and tries to transfer the logic of no customizable products to the modular products in order to support different individual needs.

The proposed methodology includes seven steps (Fig.1) and each step is characterized from several subclasses to introduce the methodology as a general framework to implement for the process of MC.

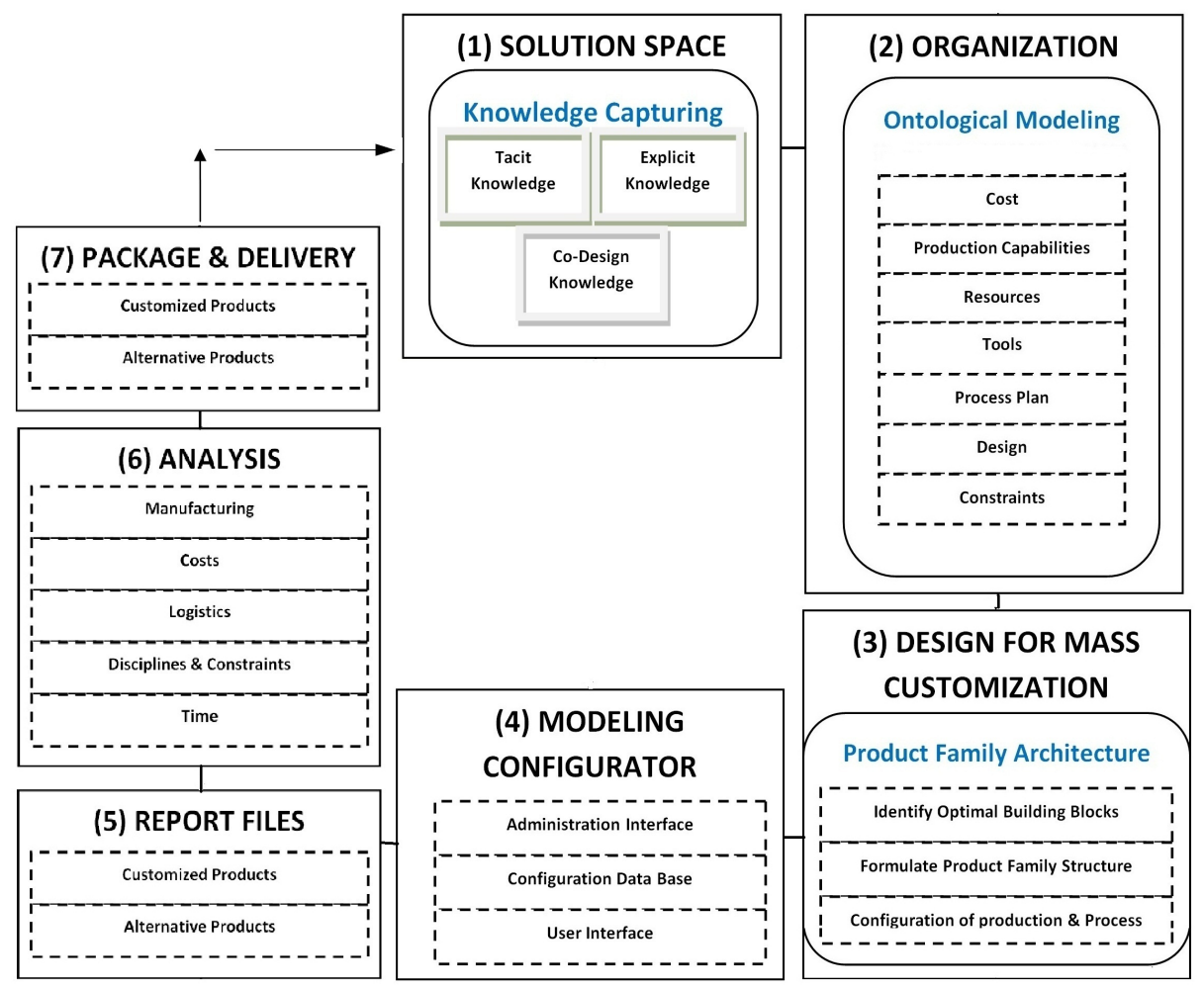

Fig. 1. Seven steps of Knowledge-Based engineering methodology in Mass Customization

The first step (Solution Space) includes the identification of the objectives, scope and assumptions about a specific project and the definition of the required knowledge sources. This phase includes three different types of knowledge: (i) tacit knowledge, (ii) explicit knowledge and (iii) co-design knowledge (the collaboration between customers and company): 
- Tacit knowledge: most of the knowledge used in the design and development process is tacit [15]. Tacit knowledge typically is developed by trial and error in practices and intuitions. Judgment, values, beliefs, assumptions and subjective insights are examples of this knowledge.

Explicit knowledge: this type of knowledge can be generally written down or otherwise documented (through print, electronic methods and other formal means), and then shared [16].

- Co-design knowledge: this knowledge is related to customer needs and co-design experiences, coming from customers' emotional connection with the product and purchasing process. Physio, Socio, Psycho and Ideo are four types of pleasure associated with products [17]. Physio pleasure related to the body and it is the feedback from sensory organs such as touch, taste, smell, etc. Socio pleasure is the result of connection with individual or groups that gives the feedback about owners view in society. Psycho pleasure is the emotional reaction result from cognitive interaction, and finally Ideo pleasure is taste, moral values and personal aspirations that define how people would like to be.

Solution Space acts as a container for these three types of knowledge, to be used in the process of MC. Each type of knowledge is extracted through the process that is using in the first three steps of MOKA methodology (Identify, Justify and Capture). In previous KBE methodologies, the knowledge container has included only tacit and explicit knowledge, while the proposed methodology adds the third co-design knowledge (Fig. 2) to consider changes in customer needs.

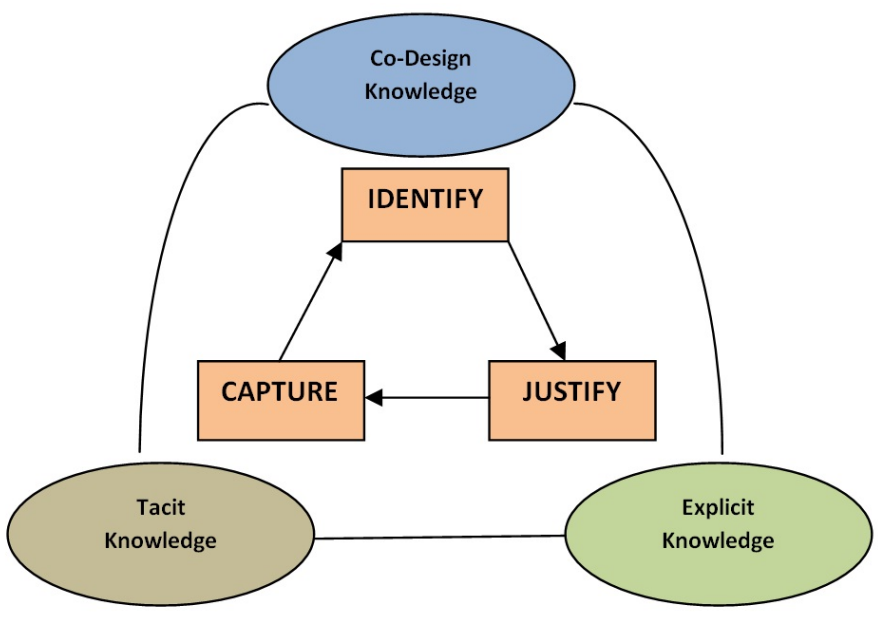

Fig. 2. Details about step one (Solution Space)

The second step (Organization) is a necessary step about knowledge utilization and organization in the process of modeling and analysis. The knowledge extracted through the first step should be provided in a way to give possibility to stakeholders to automatically access and retrieve the necessary knowledge. To do this, ontology can 
be used. Ontology forms a formalized knowledge structure through the use of concept definitions and interrelationships as expressed in axioms to support the instantiation of captured knowledge within the structure [12].

The third step is called Design for Mass Customization. To support customized product, a product family platform is required to characterize the different needs of customer by the process of Product Family Architecture (PFA). PFA means the underlying architecture of firm's product platform that various product can derived from basic product designs in order to satisfy different types of customer needs [13]. A good PFA provides a generic architecture to keep the variant forms of the same solution based on individual customer needs within a coherent framework and a good PFA development depends to the appropriate formulation of building blocks with paying attention to functional, behavioral and structural perspectives. Building block has two kinds of meaning, first a type of building blocks through modularity that means to decompose a system to modules and second for each module, various instances that shows certain similarity. Before starting to find the optimal building blocks, the information about current and future customs need should be evaluate to find the repeatability in design and fulfillment.

The fourth step of the methodology is Modeling Configurator. When a product line falls into catalog products, then a simple configurator is the best condition tool to minimize risk by accurate assessments of costs and profits about specific production before a project starts [18]. Generally, the framework of these configurators consists of an administration interface, a configuration database and an user interface. After the modeling step, the results from configurator must be published as a Report File to show the specification of finalized product in terms of technology, processes of production and functions. We can identify two kinds of report files: (i) data related to customized products(ii) data about alternative configuration models. The sixth step is the Analysis. There are different approaches to analyze the reports files and through them, DEE (Design Engineering Engine, [19]) could be a proper source for this section. The DEE methodology employs report files that contain the set of product and process models in order to use in detailed analysis modules. These modules calculate the design implications on a per-discipline basis. Manufacturability, disciplines and constraints, cost and logistics are four areas to analyze the models. The last step (Package \& Delivery) of the methodology starts with a check for the solutions about customize, alternative and pure products versus the requirements specified at the beginning of the design process. If this study is accepted, the detailed analysis results and resource implication about three kinds of products could be delivered. The first step of proposed methodology in the process of knowledge capturing is a combination with three steps of MOKA methodology and the first step of KNOMAD methodology, but the sources of knowledge are different. In addition, the second step some similarities with the Formalize step in MOKA and the third step in KNOMAD. Furthermore, the Analysis step has some similarities with the fifth and the sixth steps of KNOMAD. Finally, step seven Package \& Delivery is similar to MOKA steps Package and Activate. 


\section{Application Case}

The following application case provides a practical implementation of the proposed methodology in apparel industry. The purpose is to validate the first step of the proposed methodology through providing three kinds of knowledge related to apparel industry to deal fast as possible with changes in consumers' needs and habits. Data are collected through two approaches. From one side analyzing various configurators that are using by different companies and from other side by a survey, which was answered by users and experts. Fine Cotton, Nur Berlin, Custom Panties, Blume and Diejeans are five companies selected to extract knowledge through their configurators with some clear differentiations to represent better the concept of knowledge extraction. The specific objective of this study is to identify the fundamental variables in apparel industry and use them to develop the building blocks, which are essential for the third step of the methodology.

In apparel industry there are different features such as material, body measurement, color, size, pocket shape for each product that are acting as various blocks. Assembling of these blocks with paying attention to the importance and consequences of each block in the process of configuration gives customized products. For example, based upon the analysis of five shirt manufactures' configurators, blocks are divided in two general categories as Fit and Style and each of these categories is divided to five blocks. In order to receive the most customized products, it is very important to implement the configuration process with all blocks that are prepared in each category. By the data extracted through survey about apparel characterizes, $81 \%$ of respondents believed that size range is the first and most important constraint about cloths and as a second constrain $73 \%$ answered fabric is important for them and only $58 \%$ believed that style is important. Diversity of colors by $37 \%$, functionality $31 \%$ and weight $8 \%$ are in next grades. After this, the identified knowledge should be justified. Justification process has done paying attention to the sequence of block in terms of importance and use.

In this case, block Size is selected for justification process. One of the significant problems in apparel industry is the diversity of people sizes because of many different ethnic groups. Usually homogeneous populations are much easier to supply. For example in some countries such as Japan and Korea, only dozen sizes of people's apparel are typically needed. By comparison, people's clothing is developed and sold in categories for misses, juniors and petites with about eight to twelve sizes in each category. In addition to the issue of diversity, there are more issues such as the intermarriage and lifestyle changes, including eating habits and general fitness that producer should care about them. Product developers and buyers must be knowledgeable about the target population of the geographic area to which they market if they are to produce apparel that will fit their target customers. Sizing problem is common to all categories of clothing, including men's, women's and children's. For example in women's body measurements, the variations between the mature and general populations tended to occur only in the location and distribution of weight and shape. This separation of women body's shapes (in four domains) is the result of justification process and helps to reach accurate building blocks. 
However, with paying attention to the point that body's size depends to ethnic groups, it should precise more. The next step will solve this problem to reach the final knowledge that exactly makes the nature of building blocks. These final blocks will be uses in step Design for Mass Customization.

\section{Conclusions}

Because of the MC's nature, it is important to find important factors of customization for different industries to realize and capture knowledge from hidden market niches and subsequently develops technical capabilities to meet the different types of needs for target customers. The proposed methodology has some advantages, both in terms of $\mathrm{MC}$ and $\mathrm{KBE}$ :

- In the proposed methodology, the process will repeat in a lifecycle mood and this opportunity will facilitate to find the optimal building blocks that plays critical role in design for $\mathrm{MC}$.

- $\mathrm{MC}$ process starts with finding the customers individual needs and ending with fulfillment process targeting each particular customer. The proposed methodology also includes this important challenge about MC through step four.

- The most important step is knowledge re-use process. After the knowledge captured from internal and external sources, the knowledge will be used to model product family structure. In the process of modeling, sometime the product family will not change and this is exactly the time for reuse of previous captured knowledge.

The proposed methodology has some limitations and further researches are need:

- The validation process could be improved with more application.

- The development of the methodology should implement also other aspect of Mass Customization such as sale, cost, logistics etc.

\section{References}

1. Pine, B., Davis, S.: The New Frontier in Business Competition. Harvard Business School Press (1999) ISBN 0-87584-372-7

2. Felfernig, A.: Standardized Configuration Knowledge Representations as Technological Foundation for Mass Customization, University Klagenfurt (2007)

3. Ackoff, R.L.: From Data to Wisdom. Journal of Applies Systems Analysis 16, 3-9 (1989)

4. Ulrich, P.V., Anderson-Connell, L.J., Wu, W.: Consumer Co-Design of Apparel for Mass Customization. Journal of Fashion Marketing and Management 7, 98-412 (2003)

5. Meyer, M.H., Lehnerd, A.P.: The Power of Product Platforms, New York (1997)

6. Polanyi, M.: The logic of Tacit Inference. In: Grene, M. (ed.), pp. 138-158. University of Chicago Press, Chicago (1969)

7. Pan, S.L., Scarborough, H.: Knowledge Management in Practice, An Exploratory Case Study. Technology Analysis \& Strategic Management 11(3) (1999) 
8. Gupta, J., Sharma, S., Hsu, J.: Creating Knowledge Based Organizations (2004) ISBN 159140-162-3

9. Sandberg, M.: Knowledge Based Engineering In product Development, Department of Applied Physics and Mechanical Engineering, Lulea University of Technology (2003) ISSN 1402-1536

10. Stokes, M. (ed.): Managing Engineering Knowledge-MOKA. In: Methodology for Knowledge Based Engineering Application. ASME Press

11. Callot, M., Kneebone, S., Oldham, K., Murton, A., Brimble, R.: MOKA - A Methodology for Developing Knowledge Based Engineering Apllications (1998)

12. Curran, R., Verhagen, W.J.C., Van Tooren, M.J.L., Van der Laan, T.H.: A multidisciplinary Implementation Methodology for Knowledge-based Engineering, KNOMAD. Expert Systems with Applications, 7336-7350 (2010)

13. Kamali, N., Loker, S.: Mass customization: on-line consumer involvement in product design. Journal of ComputerMediated Communication 7(4) (2002)

14. Xuan, F.Z., Ram, D.S.: Platform-Based Product Design and Development, A Knowledge Intensive Support Approach. Manufacturing System Integration Division National Institute of Standards and Technology, Gaithersburg, MD 2089, USA (2006)

15. Blecker, T., Abdelkafi, N., Kreuter, G., Friedrich, G.: Product Configuration Systems, University of Klagenfurt, Universitätsstrasse 65-67, A 9020 Klagenfurt, Austria (2004)

16. Fulton, J., Pal, S.: Leveraging Tacit Knowledge in The New Product Development Process. ASAC, Toronto, Ontario (2005)

17. Sanchez, R.: "Tacit Knowledge" versus "Explicit Knowledge", Approaches to Knowledge Management Practice, Department of Industrial Economics and Strategy (2006)

18. Cooper, S., Fan, I., Li, G.: A Best Practice Guide - Achieving Competitive Advantage through Knowledge-based Engineering, Department of Trade and Industry, UK (1999)

19. La Rocca, G., Van Tooren, M.J.L.: Enabling Distributed Multidisciplinary Design of Complex Products, A Knowledge Based Engineering Approach. Journal of Design Research 5(3), 333-352 (2007) 